\title{
Tight-binding molecular-dynamics study of the hydrogen vibration spectrum on a $\mathrm{Si}(111)$ surface
}

\author{
B. J. Min, Y. H. Lee, ${ }^{*}$ C. Z. Wang, C. T. Chan, and K. M. Ho \\ Ames Laboratory, Microelectronics Research Center, and Department of Physics and Astronomy, \\ Iowa State University, Ames, Iowa 50011
}

(Received 6 January 1992; revised manuscript received 29 June 1992)

\begin{abstract}
The vibration spectrum of hydrogen on a $\mathrm{Si}(111)$ surface is studied by molecular-dynamics simulation using an empirical tight-binding potential model. The frequencies of the stretching and wagging modes of hydrogen obtained from the simulations are in excellent agreement with experiment. An extra mode on the hydrogen overlayer at $62 \mathrm{meV}$, which has been observed in electron-energy-loss experiments, is also reproduced. We show that the extra mode originates from the coupling between the $\mathrm{H}$ wagging mode and a $\mathrm{Si}$ substrate phonon mode. The frequency shifts of the stretching and the wagging mode of $\mathrm{H}$ with temperature are also obtained from our simulations.
\end{abstract}

\section{INTRODUCTION}

The properties of hydrogen on silicon surfaces have been studied extensively because of its importance in technology in the areas of surface passivation and amorphous semiconductors. ${ }^{1}$ In addition, hydrogen on the $\mathrm{Si}(111)$ surface $[\mathrm{Si}(111): \mathrm{H}]$ has also attracted considerable interest as a simple prototype system for studies of adsorbate vibration and diffusion on surfaces. Recent advances in surface preparation techniques ${ }^{2}$ have made it possible to fabricate clean $\mathrm{Si}(111): \mathrm{H}(1 \times 1)$ surfaces of exceptionally high quality and enable the collection of detailed experimental data with much reduced noise. The vibration spectrum has been studied extensively by high-resolution electron-energy-loss spectroscopy (HREELS), ${ }^{3,4}$ infrared spectroscopy, ${ }^{5}$ and He-atom scattering. ${ }^{6,7}$ In addition, recent experiments have also investigated the anharmonic behavior of hydrogen vibrations on the surface: Detailed measurements of the frequency shift of the hydrogen stretching mode with temperature and multiphonon processes have been performed. ${ }^{5}$ These data provide detailed information to test various models for the hydrogen-silicon interaction. On the theoretical side, the vibrational properties of the $\mathrm{Si}(111): \mathrm{H}$ system have been studied by lattice dynamics calculation ${ }^{7}$ using a bond charge model ${ }^{8}$ and by firstprinciples local-density-functional calculation using the frozen-phonon method. ${ }^{9}$ However, the finite-temperature behavior and anharmonic effects of this system still remain for the most part uninvestigated.

In this paper, we report on a molecular-dynamics (MD) simulation study of the vibrational properties of hydrogen on a $\mathrm{Si}(111)$ surface as a function of temperature. Complementary lattice dynamics calculations have also been performed to compare with the results obtained from MD simulation. In our calculations, the interatomic interactions are described by empirical tight-binding potential models ${ }^{10}$ which include the effects of covalent bonding explicitly through the electronic band structure.
Our study not only yields the vibration spectrum of the hydrogen overlayer in good agreement with experimental data, but also provides detailed information on the hydrogen-silicon interactions in this system. The rest of the paper is arranged as follows: In Sec. II, a brief description of the empirical tight-binding model used in our MD study is presented. In Sec. III, the vibration spectrum of $\mathrm{H}$ on the $\mathrm{Si}(111)$ surface from the MD simulation is presented. In Sec. IV, results on the anharmonic effects are discussed.

\section{EMPIRICAL TIGHT-BINDING MODEL}

The Hamiltonian governing the atomic motion in the MD simulation is

$$
\begin{aligned}
H= & \Sigma \frac{P^{2}}{2 M_{\mathrm{Si}}}+\Sigma \frac{p^{2}}{2 m_{\mathrm{H}}}+\Sigma_{n} \epsilon_{n}+\frac{1}{2} \Sigma^{\prime} V_{\mathrm{Si}-\mathrm{Si}}\left(\mathbf{R}_{i}, \mathbf{R}_{j}\right) \\
& +\frac{1}{2} \Sigma V_{\mathrm{Si}-\mathrm{H}}\left(\mathbf{R}_{i}, \mathbf{r}_{j}\right),
\end{aligned}
$$

where the first two terms are the kinetic energies of the $\mathrm{Si}$ and $\mathrm{H}$ ions, respectively. The third term is the electronic band energy calculated through a nearest-neighbor empirical tight-binding description of the $\mathrm{Si}(111)$ : $\mathrm{H}$ system; the last two terms are short-ranged repulsive pair interactions between $\mathrm{Si}$ atoms and between $\mathrm{Si}$ and $\mathrm{H}$. The interactions between $\mathrm{H}$ atoms are neglected here since the hydrogen atoms stay far away from each other in our simulations. The parameters for the tight-binding model and the pair potentials are given in our previous papers. ${ }^{10,11}$ Our model reproduces various properties of the $\mathrm{Si}(111): \mathrm{H}$ surface in comparison to first-principles local-densityapproximation (LDA) calculations: It yields $74 \mathrm{meV}$ for the wagging mode of the $\mathrm{Si}(111): \mathrm{H}$ surface at $T=0$ in a frozen-phonon calculation in which the energy of the crystal is calculated as the hydrogen position is varied. The corresponding LDA calculation yields a result of 71 meV. ${ }^{9}$ The stretching-mode frequency in our model is $275 \mathrm{meV}$ compared to a LDA frozen-phonon result of 
$245 \mathrm{meV} .{ }^{9}$ The measured frequencies for the stretching and wagging mode are 257 and $77 \mathrm{meV}$, respectively. ${ }^{12}$

\section{VIBRATION SPECTRUM OF Si(111):H}

The MD simulation is performed for a $\mathrm{Si}(111): \mathrm{H}$ slab consisting of six layers of $\mathrm{Si}$ and two layers of $\mathrm{H}$ attached to each $\mathrm{Si}(111)$ surface and with nine atoms on each layer. Periodic boundary conditions are imposed in the directions parallel to the surface. The simulation temperatures range from 200 to $600 \mathrm{~K}$. The time step $\Delta t$ is taken as $2.7055 \times 10^{-16} \mathrm{sec}$. One interesting feature of the present problem is that, because of the light mass of the hydrogen atom, the zero-point energy of the $\mathrm{H}$ Si stretching mode has a value much larger than $k_{B} T$ in the temperature range for the simulations. In our calculations, we follow a procedure in which the zeropoint energy of the stretching mode is deposited to the hydrogen layer by uniformly stretching the $\mathrm{Si}-\mathrm{H}$ bonds normal to the surface at the beginning of the simulation. When the system temperature is raised up, the temperature control is imposed only on those Si layers which are not directly connected to the hydrogen layers. More discussions on this subject will be presented in the next section. At each temperature, the system is allowed to equilibrate for more than $10000 \mathrm{MD}$ steps. After the equilibration of the system, the trajectory of the following $65536 \mathrm{MD}$ steps (corresponding to a total time interval of $17.7 \times 10^{-12} \mathrm{sec}$ ) is collected for analysis. The phonon spectrum is obtained by taking the Fourier transform of the velocity-velocity autocorrelation function.

$$
G(\mathbf{k}, \omega)=\int d t e^{i \omega t} \sum_{n} e^{-i \mathbf{k} \cdot \mathbf{R}_{n}} \frac{\left\langle\mathbf{v}_{n}(t) \cdot \mathbf{v}_{0}(0)\right\rangle}{\left\langle\mathbf{v}_{n}(0) \cdot \mathbf{v}_{0}(0)\right\rangle}
$$

In this formulation, the phonon spectrum for each layer of the slab can be obtained by taking the velocities of the particles that belong to that specific layer only.

To compare with the results from MD simulations, we have also calculated the phonon spectrum at $T=0 \mathrm{~K}$ by lattice-dynamical method. In the lattice dynamics calculation, the force-constant matrix $K$ is determined by calculating the force $F$ on each atom for small displacements $x$ of each atom in the six-layer unit cell,

$F_{i \alpha}=-K_{i \alpha, j \beta} x_{j \beta}, i, j=1,2, \ldots, N, \alpha, \beta=1,2,3$.

The normal modes of the system can be determined from the eigenvalue equation:

$$
\operatorname{det}\left(K_{i \alpha, j \beta} x_{j \beta}-M_{i} \omega^{2} x_{i \alpha}\right)=0 .
$$

We consider a six-layer unit cell with the $\mathbf{k}$-point sampling equivalent to the $3 \times 3 \times 6 \mathrm{MD}$ cell to calculate the Hellmann-Feynman force arising from the band-structure energy.

In Fig. 1, the MD simulation results for the phonon spectrum of the hydrogen overlayer (on top) and the silicon substrate layers at $300 \mathrm{~K}$ (MD temperature) are presented. The spectrum at other temperatures (200-600 K) are very similar to that of Fig. 1 apart from a slight shift in the frequency and a broadening in the peak width which will be the subject of discussion in the next section.

The results of the lattice dynamics, i.e., the magnitude of the normal mode at the specific layer from the latticedynamics calculation, is also plotted against the normal mode frequency in Fig. 2.

On the hydrogen overlayer, three strong peaks have been observed at positions of 267,77 , and $61 \mathrm{meV}$ from $\mathrm{MD}$ and 275, 78, and $62 \mathrm{meV}$ from lattice dynamics. The first two modes can be identified as the stretching and wagging modes of $\mathrm{Si}-\mathrm{H}$. The frequencies of these two modes obtained from the MD simulation are in excellent agreement with the experimental data of 257 and 77 $\mathrm{meV}$, respectively. The difference between the MD result and lattice dynamics result of the frequency of the stretching mode is due to the different vibration amplitudes used in the two calculations. In the latticedynamics calculation, a small vibration amplitude $(\sim$ $0.01 \AA$ ) has been used, yielding the stretching mode frequency comparable to that obtained by the frozenphonon calculation, whereas in the MD simulation, the

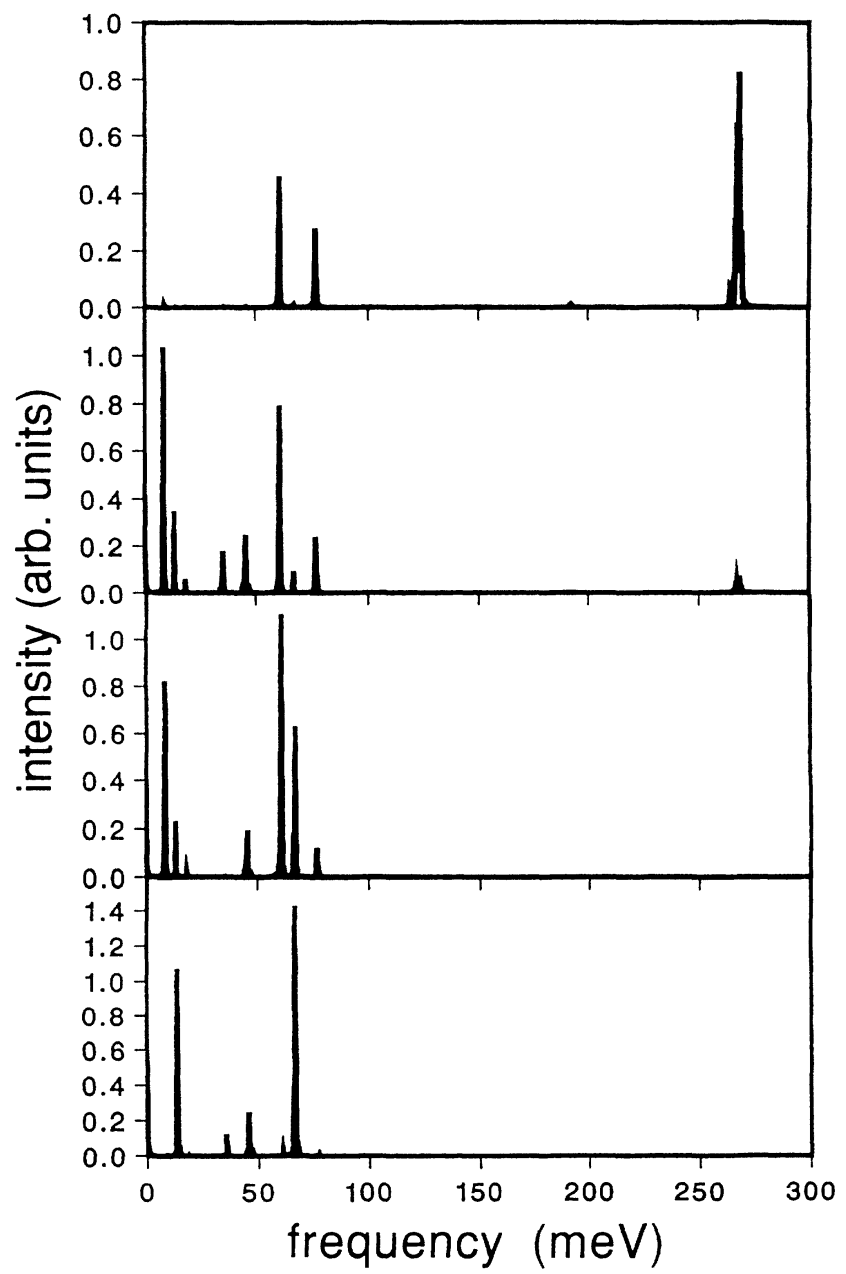

FIG. 1. Phonon spectral intensity for the atoms of $\mathrm{H}$ overlayer, first $\mathrm{Si}$ layer, second $\mathrm{Si}$ layer, and third $\mathrm{Si}$ layer from the top, respectively, calculated from the MD simulation. 


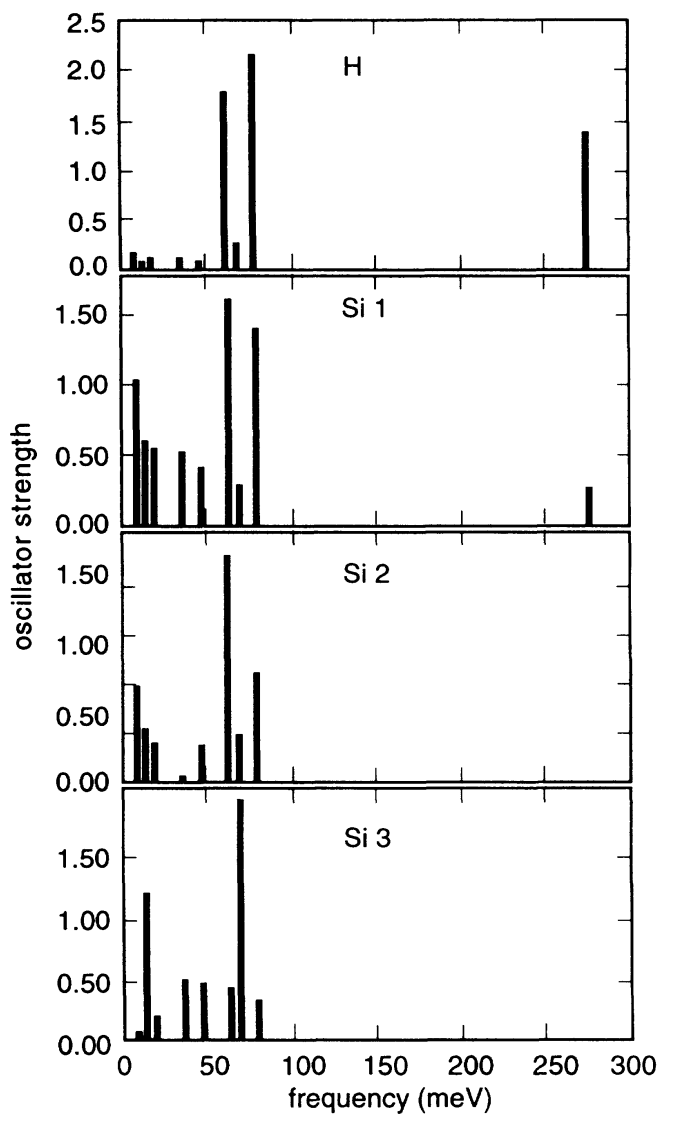

FIG. 2. Normal mode strengths at the surface from the lattice-dynamics calculation of $\operatorname{Si}(111): \mathrm{H}$.

calculation is performed for a larger vibration amplitude $(\sim 0.1 \AA)$ for the stretching mode corresponding to the zero-point energy of the Si-H bond. Additional temperature effects are small and usually cause a slight downward shift in the phonon frequencies.

The most notable feature of the vibrational spectrum of the hydrogen overlayer is that an extra peak appears at $61 \mathrm{meV}$. Also, the frequency of the wagging mode (77 $\mathrm{meV}$ ) is higher than the frozen-phonon result of $74 \mathrm{meV}$. It appears that the polarization of the extra mode is entirely in-plane and propagates into the Si substrate layers with its intensity getting higher and its position shifting towards $67 \mathrm{meV}$. This feature suggests that this extra mode is in fact a surface resonance. The extra mode at $62 \mathrm{meV}$ in addition to stretching and wagging modes was first noted by Froitzheim, Lammering, and Günter ${ }^{3}$ from the off-specular HREELS spectrum of the $\mathrm{Si}(111): \mathrm{H}$ surface. Originally, they have assigned this mode to vibrations of Si: $\mathrm{H}_{3}$ species present on the surface. Further investigations by the same authors showed that $\mathrm{Si}: \mathrm{H}_{3}$ is not present on the surface, leading to the speculation that this extra loss is due to a substrate mode induced by the adsorbate and possibly coupled to the bulk phonons of $\mathrm{Si}^{4}$ The lattice-dynamics calculation results of Harten $e t$ $a l .{ }^{7}$ also confirmed that the extra mode around $61 \mathrm{meV}$ is indeed an intrinsic property of the $\mathrm{Si}(111): \mathrm{H}$ system.

In order to understand the origin of this extra mode, we replace all $\mathrm{H}$ atoms by deuterium (D) atoms and repeat the lattice-dynamics calculation. The vibration spectrum of the $\mathrm{Si}(111): \mathrm{D}$ system obtained by this calculation is shown in Fig. 3. We also observe three major peaks in the D layer at 49,70 , and $197 \mathrm{meV}$. Contrary to the case of $\mathrm{Si}(111): \mathrm{H}$, the frequency of the wagging mode of $\mathrm{D}(49 \mathrm{meV})$ is now smaller than the value obtained by the frozen-phonon calculation $(52 \mathrm{meV})$. In addition, the position of the extra peak at $70 \mathrm{meV}$ is higher than that of the corresponding peak (at $62 \mathrm{meV}$ ) in the hydrogen overlayer.

The origin of the extra peak or surface resonance on the overlayer can be explained by considering the coupling between the wagging mode of $\mathrm{SiH}$ (or $\mathrm{SiD}$ ) and a substrate mode originating from the $\mathrm{TO}(\Gamma)$ mode of bulk $\mathrm{Si}$. On the $\mathrm{Si}(111)(1 \times 1)$ termination, the $\mathrm{TO}(\Gamma)$ mode has uniform in-plane polarization and can couple to the wagging modes of the overlayer if their energies are close enough. From a frozen-phonon calculation, we found that the bare frequency of the TO $(\Gamma)$ mode (without coupling to the overlayer) is $68 \mathrm{meV}$ in our model, which is quite close to the bare wagging frequency of the $\mathrm{SiH}(74 \mathrm{meV})$ and of the $\mathrm{SiD}(52 \mathrm{meV})$. As the coupling is turned on, we expect the frequencies would shift as illustrated in Fig. 4. In the $\mathrm{Si}(111): \mathrm{H}$ system, the frequency of the wagging mode will shift up and the fre-

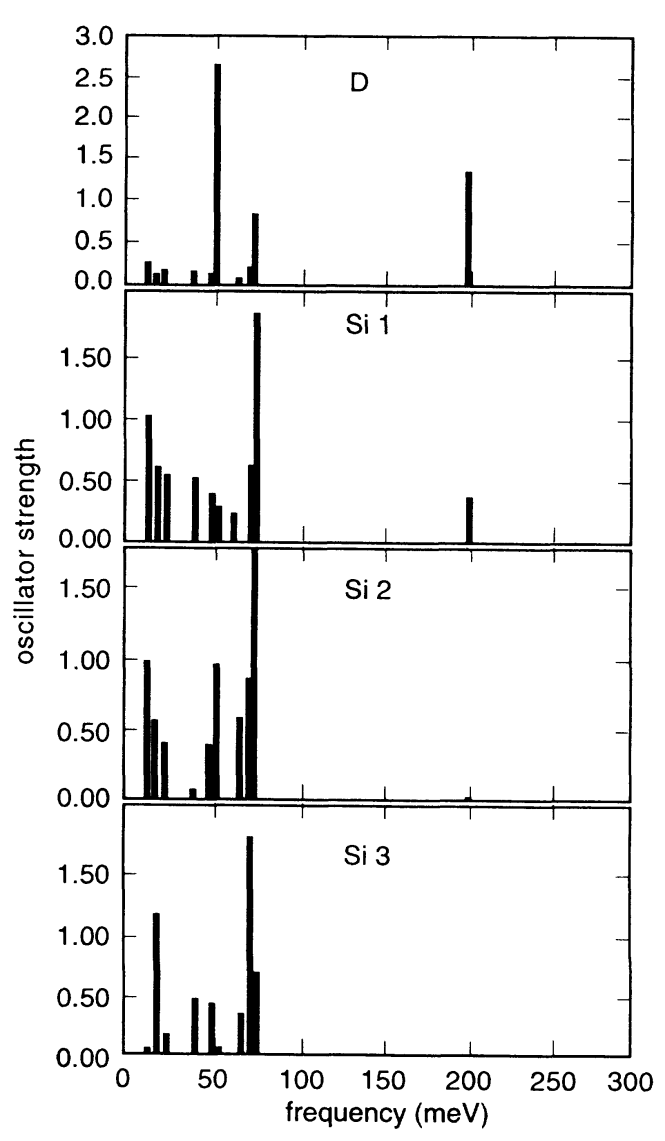

FIG. 3. Normal mode strengths at the surface from the lattice-dynamics calculation of $\mathrm{Si}(111): \mathrm{D}$. 

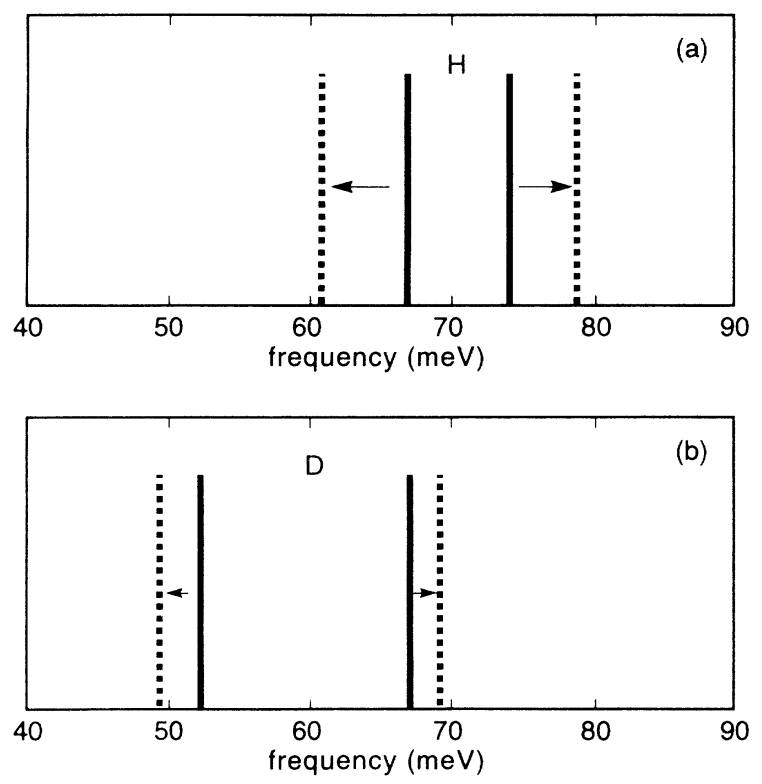

FIG. 4. Schematic representation of the frequency shift caused by the coupling between the wagging mode and the $\mathrm{Si}$ substrate phonon from the lattice-dynamics calculation. On top, $\mathrm{H}$ wagging mode $(74 \mathrm{meV})$ interacts with $\mathrm{Si}$ substrate mode $(68 \mathrm{meV})$. On bottom, D wagging mode $(52 \mathrm{meV})$ interacts with the same $\mathrm{Si}$ substrate mode $(68 \mathrm{meV})$ resulting in the frequency shift in the opposite direction.

quency of the surface resonance will shift down from their bare frequencies, respectively, while in the $\mathrm{Si}(111)$ : $\mathrm{D}$ system, the shift is in the opposite direction since the bare frequency of the wagging mode is lower than that of the $\mathrm{TO}(\Gamma)$ mode. We expect the frequency shifts of the wagging and the $\mathrm{TO}(\Gamma)$ modes should be larger in the case of $\mathrm{Si}(111): \mathrm{H}$ and smaller in the case of $\mathrm{Si}(111): \mathrm{D}$ since in the former case the two bare frequencies are much closer to one another. This analysis is consistent with our lattice-dynamics calculation and MD simulation results, and also with the experimental results. ${ }^{4}$

\section{ANHARMONIC EFFECTS}

From the simulations, we can also obtain the frequency shift of the stretching mode as a function of the Si substrate temperature. Because of the small mass of the hydrogen atom, we are in the regime where the zero-point energy is larger than $k_{B} T$ and quantum-mechanical effects are important. Since MD simulation is based on Newton's equations of motion following the rules of classical statistical mechanics, it would be interesting to see what happens when one applies a classical scheme in this regime. When we performed the MD simulation on this system in the conventional way as we did for the pure $\mathrm{Si}$ system, ${ }^{11}$ we obtained a very large frequency shift with temperature (approximately ten times bigger than the experimentally observed shift) and a much broader linewidth (approximately $4 \mathrm{meV}$ ) for the hydrogen stretching mode. This discrepancy arises from the large difference between the classical and quantum results for the hydrogen kinetic energy distribution in the quantum regime. When the zero-point energy for the stretching mode is quite a bit bigger than $k_{B} T$ over the range of simulation temperatures, most of the hydrogen will be in the ground-state energy level. Thus, the distribution of vibration amplitudes for the hydrogen vibrations is much narrower than the classical Boltzmann distribution assumed in the MD simulation. Furthermore, the quantum-mechanical distribution remains relatively unchanged over the range of simulation temperatures whereas the classical distribution is much more temperature sensitive. This difference causes exaggerated temperature dependence of the hydrogen vibration properties. While a quantum-mechanical simulation of the hydrogen motion is still a difficult task at present, we tried to simulate the quantum hydrogen stretching-mode amplitude distribution in the present study by depositing an energy equivalent to the zero-point energy of the stretching mode in each $\mathrm{Si}-\mathrm{H}$ bond at the start of each MD simulation. This is done by uniformly stretching the $\mathrm{Si}-\mathrm{H}$ bonds normal to the surface. When the system is heated up, the temperature control is imposed on those Si layers which are not directly connected to the hydrogen layers. Since the frequency of the stretching mode of $\mathrm{H}$ is well separated from that of any other phonon modes in the system, the energy in the stretching mode stays fairly constant at the zero-point energy. ${ }^{13}$ The temperature effects on the frequency shift and linewidth for this mode are mediated through the temperature dependence of the atomic motions of the Si layers. We find that this ad hoc procedure yields more reasonable results for the frequency shifts and phonon linewidths. The typical phonon spectral densities are shown in Figs. 5 and 6 , after smoothing with a Lorentzian of width $0.5 \mathrm{meV}$.

For comparison with the experiment, we relate the average kinetic energy $(\langle\mathcal{K}\rangle)$ of the $\mathrm{Si}$ atoms in the $\mathrm{MD}$ simulation to the experimental temperature $(T)$ by

$$
\langle\mathcal{K}\rangle=\frac{1}{2} \int h \nu D(\nu)\left[\frac{1}{2}+\frac{1}{e^{h \nu / k_{B} T}-1}\right] d \nu,
$$

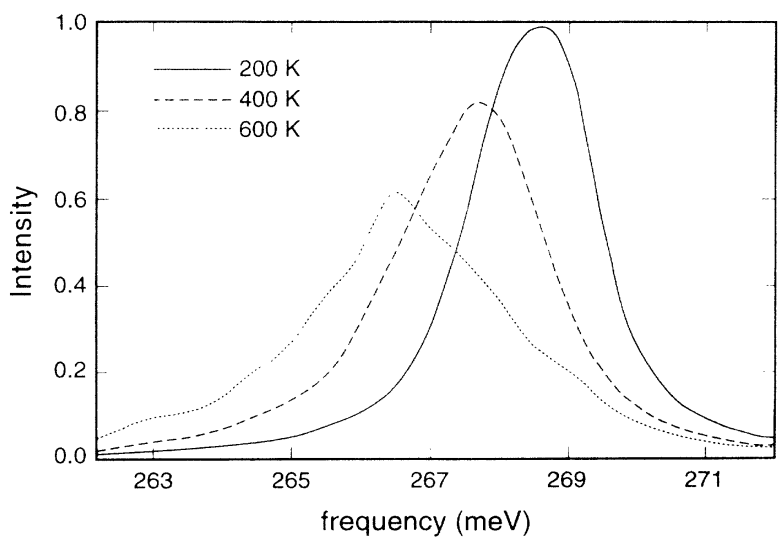

FIG. 5. Phonon spectral intensity of the hydrogen stretching mode at $T_{\mathrm{MD}}=200,400$, and $600 \mathrm{~K}$ from the MD simulation. 


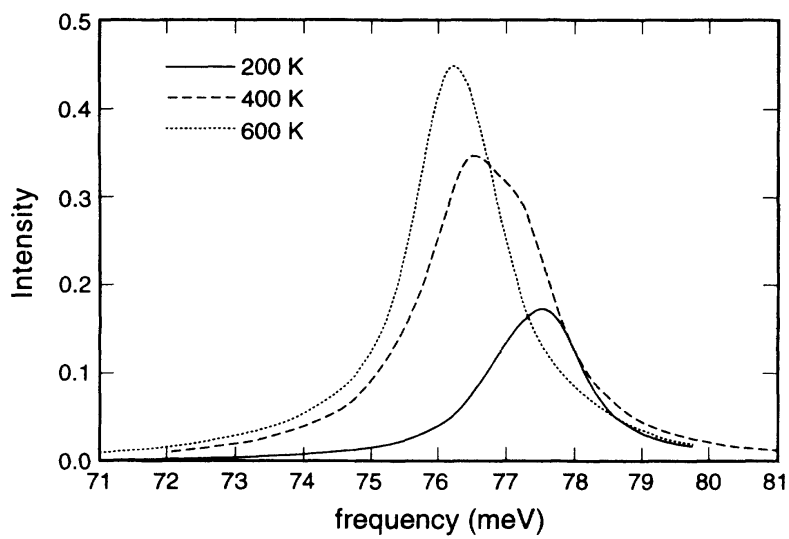

FIG. 6. Phonon spectral intensity of the hydrogen wagging mode at $T_{\mathrm{MD}}=200,400$, and $600 \mathrm{~K}$ from the MD simulation.

where $D(\nu)$ is the phonon density of states of the $\mathrm{Si}$ substrate, instead of using the usual classical MD temperature $\left(T_{\mathrm{MD}}\right)$ obtained from the equipartition result $\langle\mathcal{K}\rangle=\frac{3}{2} k_{B} T_{\mathrm{MD}}$ per atom. In our previous studies of anharmonic phonon effects in bulk silicon and diamond, ${ }^{11}$ we found that this procedure gives a good description of the anharmonic effects due to the zero-point motion of the silicon atoms at low temperatures. The results are plotted in comparison with experiment ${ }^{5}$ in Fig. 7. The frequency shift is larger by a factor of about $1.5 \mathrm{com}$ pared to the experiment. This deviation may be due to the strong quantum nature of the hydrogen motion which cannot be simulated properly by classical MD. The calculated linewidth of $1.0 \mathrm{meV}$ is broader than the experimentally observed linewidth (about $0.1 \mathrm{meV}$ at $300 \mathrm{~K}$ ). However, when we calculate the velocity autocorrelation function of one $\mathrm{H}$ atom, then the linewidth is approximately $0.25 \mathrm{meV}$. The fact that we obtained a smaller linewidth for the motion of one atom than for the phonon indicates that the stretching mode is basically dispersionless. It also indicates that the wide linewidth observed

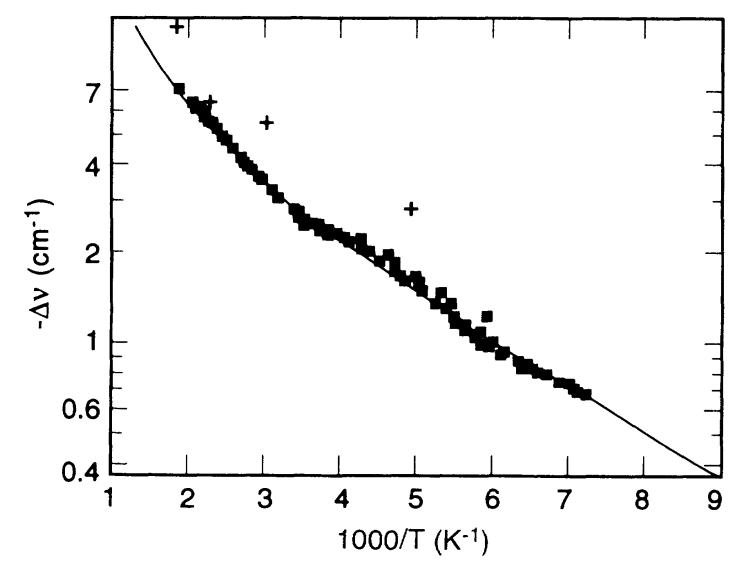

FIG. 7. The frequency shift from the MD simulation (marked with + ) compared with the experiment. in the phonon velocity autocorrelation originates from inhomogeneous broadening coming from the distribution of the frequency positions of the various single-atom peaks. Since we observed no surface reconstruction in our MD simulation even at the lowest temperatures, the spread in the vibration frequencies of different $\mathrm{H}$ atoms arises from a spread in the $\mathrm{H}$ vibrational energy distribution arising from the sensitivity of the $\mathrm{H}$ vibrational energy deposited to the instantaneous thermal motion of $\mathrm{Si}$ atoms in the initial stage of the simulation during the thermalization process. Since our total simulation time is only $17 \mathrm{ps,} \mathrm{a}$ considerable part of the linewidth for the single-atom velocity autocorrelation spectrum is due to inadequate frequency resolution. Indeed, experiment ${ }^{14}$ indicates that the dephasing time for the $\mathrm{H}$ stretching mode can be as long as $30 \mathrm{ps}$ at low temperatures. Thus, we would need a much longer simulation time to observe such a linewidth. We also observed the frequency shift for the wagging mode; the results are summarized in Table I.

Recent experiments observed side peaks close to the stretching peak causing a loss in the intensity of the fundamental peak. ${ }^{5,15}$ We also found side peaks in the velocity autocorrelation spectrum for the stretching-mode vibration at frequencies $\nu_{\text {str }} \pm \nu_{\text {wag }}$ and $\nu_{\text {str }}-2 \nu_{\text {wag }}$ (Fig. 8). The side peaks at $\nu_{\text {str }} \pm \nu_{\text {wag }}$ have polarization parallel to the surface, while the side peak at $\nu_{\mathrm{str}}-2 \nu_{\mathrm{wag}}$ is perpendicular to the surface. This indicates the existence of strong anharmonic coupling between the stretching and the wagging modes. The sidepeaks at $\nu_{\text {str }} \pm \nu_{\text {wag }}$ have not been observed by experiment yet. These side peaks grow in intensity as the substrate temperature is raised but a quantitative analysis of the peak intensities could

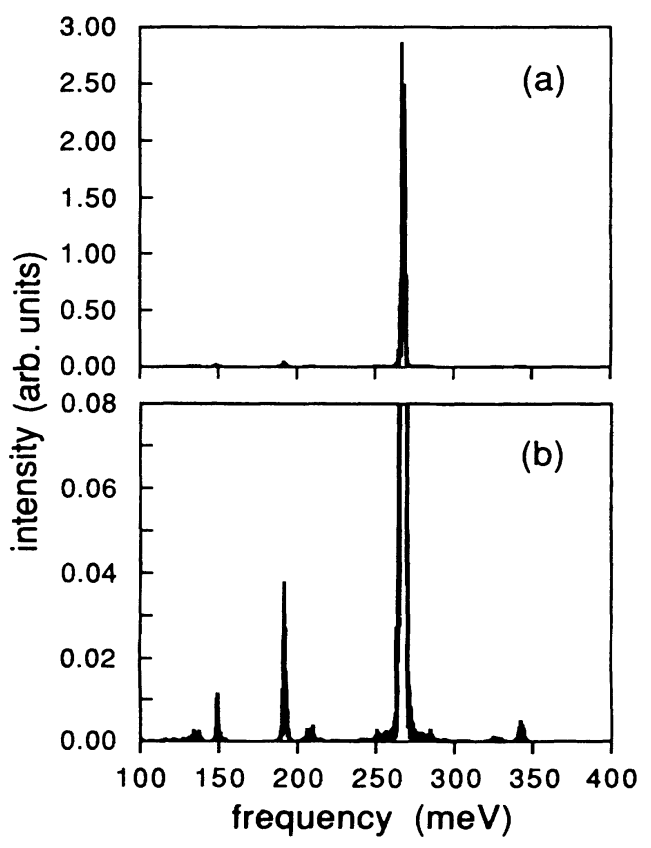

FIG. 8. Phonon spectral intensity at $T_{\mathrm{MD}}=300 \mathrm{~K}$ showing side peaks to the $\mathrm{H}$ stretching mode. Note that the vertical scales of (a) and (b) are different. 
TABLE I. The frequency (in $\mathrm{meV}$ ) of the hydrogen stretching mode and wagging mode vs silicon substrate temperature.

\begin{tabular}{ccc}
\hline \hline$T$ & Wagging mode & Stretching mode \\
\hline 0 & $77.19 \pm 0.05$ & $268.24 \pm 0.16$ \\
210 & $76.92 \pm 0.07$ & $267.92 \pm 0.23$ \\
340 & $76.57 \pm 0.10$ & $267.51 \pm 0.07$ \\
450 & $76.69 \pm 0.15$ & $267.25 \pm 0.17$ \\
560 & $76.17 \pm 0.06$ & $266.56 \pm 0.27$ \\
\hline \hline
\end{tabular}

not be made due to large fluctuations in the intensities. For a quantitative analysis, much longer simulation times would be required.

\section{SUMMARY}

To summarize, we have presented a model that reproduces well the behavior of hydrogen on the $\operatorname{Si}(111)$ surface. The existence of an additional hydrogen mode observed by EELS experiment is explained in our model by the coupling of the hydrogen wagging mode with a bulk phonon mode in Si. This coupling is responsible for the anomalous isotope shift of the hydrogen wagging mode frequencies on this surface. The calculated frequency shift and the linewidth of the stretching mode are larger than the experiment. It is likely that a quantummechanical treatment of hydrogen may be necessary to achieve a fully satisfactory description of these anharmonic effects.

\section{ACKNOWLEDGMENTS}

Ames Laboratory is operated by Iowa State University for the U.S. Department of Energy under Contract No. W-7405-ENG-82. This research was supported by the Director for Energy Research, Division of Material Sciences, U.S. Department of Energy, and by the National Science Foundation under Grant No. DMR-8819379. One of us (Y.H.L.) would like to thank the Ames Laboratory and Microelectronics Research Center for hospitality, and support from Korea Science and Engineer Foundation, and Ministry of Education of Korea.
*Permanent address: Department of Physics, Jeonbug National University, Jeonju, Jeonbug 560-756, Korea.

${ }^{1}$ For example, see the review article by S. J. Pearton, J. W. Corbett, and T. S. Shi, Appl. Phys. A 43, 153 (1987).

${ }^{2}$ H. Ubara, T. Imura, and A. Hiraki, Solid State Commun. 50, 673 (1984); M. Grundner and H. Jacob, Appl. Phys. A 39, 73 (1986); E. Yablonovitch, D. L. Allara, C. C. Chang, T. Gmitter, and T. B. Bright, Phys. Rev. Lett. 57, 249 (1986); G. S. Higashi, Y. J. Chabal, G. W. Trucks, and K. Raghavachari, Appl. Phys. Lett. 56, 656 (1990).

${ }^{3}$ H. Froitzheim, H. Lammering, and H.-L. Günter, Phys. Rev. B 27, 2278 (1983).

${ }^{4}$ H. Froitzheim, U. Köhler, and H. Lammering, Surf. Sci. 149, 537 (1985).

${ }^{5}$ P. Dumas, Y. J. Chabal, and G. S. Higashi, Phys. Rev. Lett. 65, 1124 (1990); P. Guyot-Sionnest, P. Dumas, Y. J. Chabal, and G. S. Higashi, ibid. 64, 2156 (1990); P. GuyotSionnest, ibid. 66, 1489 (1991).

${ }^{6}$ R. B. Doak, Y. J. Chabal, G. S. Higashi, and P. Dumas, J. Electron Spectrosc. Relat. Phenom. 54/55, 291 (1990).

${ }^{7}$ U. Harten, J. P. Toennies, Ch. Wöll, L. Miglio, P. Ruggerone, L. Colombo, and G. Benedek, Phys. Rev. B 38, 3305 (1988).

${ }^{8}$ J. C. Phillips, Phys. Rev. 166, 832 (1968); R. M. Martin, ibid. 186, 871 (1969); W. Weber, Phys. Rev. B 15, 4789 (1977).

${ }^{9}$ E. Kaxiras and J. D. Joannopoulos, Phys. Rev. B 37, 8842 (1988).

${ }^{10}$ B. J. Min, Y. H. Lee, C. Z. Wang, C. T. Chan, and K. M. Ho, Phys. Rev. B 45, 6839 (1992).

${ }^{11}$ C. Z. Wang, C. T. Chan, and K. M. Ho, Phys. Rev. B 39, 8586 (1989); 42, 11276 (1990).

${ }^{12}$ See a review article by M. Cardona, Phys. Status Solidi B 118, 463 (1983).

${ }^{13}$ Experimental measurements for the lifetime of the $\mathrm{Si}-\mathrm{H}$ stretching mode indicated that the time scale for leakage of energy from the Si-H mode is of the order of $0.5-1.4$ ns (Ref. 14). In our simulation, we also observed that any spread in the initial vibrational energy deposited in the $\mathrm{Si}-\mathrm{H}$ bond stretching mode persists unaltered during the duration of the simulation. This indicates that the energy transfer and equilibration process takes a time much longer than the simulation period in our calculation.

${ }^{14}$ P. Guyot-Sionnest, P. Dumas, and Y. J. Chabal, J. Electron Spectrosc. Relat. Phenom. 54/55, 27 (1990).

${ }^{15}$ P. Dumas, Y. J. Chabal, and G. S. Higashi, J. Electron Spectrosc. Relat. Phenom. 54/55, 103 (1990). 